\title{
INFLUÊNCIA DA ÉPOCA DE CONTROLE DAS PLANTAS DANINHAS, SOBRE A EFICÁCIA DO HERBICIDA ACETOCHLOR EM MILHO. Gustavo Martini $^{1}$; Aimar Francisco Ferrari Pedrinho Junior ${ }^{1}$ e Julio Cezar Durigan ${ }^{2}$
}

1. Graduando em Agronomia na FCAV/UNESP - Jaboticabal (SP), CEP 14870-000

2. Prof. Titular no Departamento de Fitossanidade da FCAV/UNESP - Jaboticabal (SP), CEP 14870-000

\section{RESUMO}

O presente trabalho objetivou avaliar a eficácia do acetochlor, aplicado num estágio precoce e semi-tardio, no controle de Cenchrus echinatus, Digitaria horizontalis, Indigofera hirsuta, Portulaca oleracea e Ipomoea grandifolia, além de verificar possíveis efeitos de intoxicação às plantas de milho. Utilizou-se uma área da Fazenda Experimental de Ensino e Pesquisa da Faculdade de Ciências Agrárias e Veterinárias, Câmpus de Jaboticabal-SP, onde cultivou-se o híbrido AG 3010, para a instalação do experimento. Testou-se três dosagens $(1,68 ; 2,52$ e $3,36 \mathrm{~kg} / \mathrm{ha})$ do acetochlor em misturas com o nicosulfuron a $0,032 \mathrm{~kg} / \mathrm{ha}$. Utilizou-se como referências para comparações entre os resultados os tratamentos com nicosulfuron isolado $(0,032 \mathrm{~kg} / \mathrm{ha})$ e as testemunhas capinada e sem capina. Os herbicidas foram aplicados com o auxílio de um pulverizador costal manual à pressão constante (mantida por $\mathrm{CO}_{2}$ comprimido) de $2,8 \mathrm{~kg} / \mathrm{cm}^{2}$, munido de barra com 4 bicos XR 110.02 e com um consumo de calda equivalente a $200 \mathrm{~L} / \mathrm{ha}$, quando as plantas daninhas apresentavam 2 folhas e mais tardiamente com 4 a 6 folhas. Deve-se preconizar a aplicação dos herbicidas mais precocemente, sendo que o acetochlor adicionado ao nicosulfuron proporcionou um melhor efeito residual, exceto para o C. echinatus onde a aplicação feita mais tardiamente proporcionou um melhor controle. As dosagens testadas do acetochlor não apresentaram diferenças expressivas de controle entre si, e podem ser utilizadas sem restrições no híbrido testado.

Palavras-chave: herbicidas, mistura em tanque, milho, pós-emergência.

\section{EFFICACY OF ACETOCHLOR APPLIED IN POST EMERGENCY ON THE WEEDS IN MAIZE.} ABSTRACT

It intend with experiment evaluate the performance of the acetochlor herbicide by itself tank mix with nicosulfuron, applied in post emergency on the following weeds: Cenchrus echinatus, Digitaria horizontalis, Indigofera hirsuta, Portulaca oleracea, Ipomoea grandifolia. The experiment was conducted at the FCAV-UNESP CAMPUS, in Jaboticabal $\mathrm{SP}$, on red oxissol. Late on, it was evaluated the possible toxicity symptoms on the maize. The treatments were: acetochlor + nicosulfuron a 1,68 +0,032;2,52+0,032 and 3,36+0,032 $\mathrm{Kg} / \mathrm{ha}$, nicosulfuron a $0,032 \mathrm{Kg} / \mathrm{ha}$, in two sprayer age, compered of the witness with and without weeds. The weeds ages application were early (2 leaf) and late (4 to 6 leaf) post emergency. The herbicides were applied with costal sprayer, to constant pressure (40 $\mathrm{lbf} / \mathrm{pol}^{2}$ ), with four nozzles XR 11002. The equivalent spray volume was $200 \mathrm{~L} / \mathrm{ha}$, and the maize used was $\mathrm{Ag} 3010$. To the $C$. echinatus the mixture acetochlor + nicosulfuron, in all the rates tested, can are applied latiest, why earned with residual. But to the other weeds evaluated, have to accomplish the herbicides aplication the earliest, and but acetochlor + nicosulfuron mixture have best residual effect. Haven't diference to control between smaller rate $(1,68+0,032)$ and larger rate $(3,36+0,032)$ at mixture. Despite the slightely reduction in 
the beginnig on maize treated with acetochlor, they recovered quikly making possible the utilization of the acetochlor on the tested hybrid.

Key words: herbicides, tank mixture, maize, post emergency.

\section{INTRODUÇÃO}

O Brasil possui condições edafocimáticas favoráveis ao cultivo do milho (Carvalho et al., 2001), ocupando, atualmente, o terceiro lugar na produção mundial (Agrianual, 2000). No entanto, um dos principais problemas é a interferência que as plantas daninhas podem causar a essa cultura, acarretando perdas de até $85 \%$ na produção de grãos, segundo Blanco (1985).

No entanto deve-se ressaltar que o uso mais intensivo dos herbicidas, como único método de controle das plantas daninhas em algumas áreas, segundo Monquero et al. (2001) ocasionam o aparecimento de populações compostas por biótipos resistentes a esses produtos, devido a elevada pressão de seleção a que são expostas. Os mesmos autores citam ainda os herbicidas que possuem grande especificidade no local de ação, com prolongado efeito residual favorecem essa pressão de seleção.

É necessário a adoção de práticas agrícolas que previnam ou retardem esse processo. Algumas são bastante simples como a rotação de culturas, que permite o uso de outros herbicidas alternativos, com diferentes mecanismos de ação. No entanto, Christoffoleti et al. (1994) ressaltam a importância do seu uso racional, evitando-se o uso de herbicidas com prolongado efeito residual e aplicações seguidas com herbicidas de mesmo mecanismo de ação.

As misturas de herbicidas constituem uma outra alternativa na prevenção de resistências das plantas daninhas, onde herbicidas com mecanismos de ação diferentes controlam eficientemente os biótipos suscetível e resistente a um dos herbicidas. No entanto as misturas comerciais prontas ou feitas no tanque momentos antes da aplicação, podem ser consideradas como fortes aliadas para evitar-se o aparecimento de resistência, por controlar um maior espectro de plantas daninhas.

Outros aliados a esse propósito são estudos de eficácia e seletividade para o milho de moléculas utilizadas em outras culturas, ampliando o leque de alternativas no controle das plantas daninhas. O acetochlor, pertencente ao grupo químico das cloroacetamidas, classificado segundo Gazziero et al. (2001) como herbicida inibidor da divisão celular da parte aérea e utilizado no controle de plantas daninhas em soja, é uma das alternativas.

Objetivou-se a avaliar a eficácia da mistura em tanque do herbicida acetochlor com nicosulfuron, comparado com o nicosulfuron isolado, aplicados em pós-emergência precoce e semi-tardia, para as principais espécies de plantas daninhas de ocorrência na área experimental. Avaliou-se também as possíveis alterações morfológicas nas plantas de milho, que pudessem ser caracterizadas como sintomas de fitointoxicação dos herbicidas.

\section{MATERIAIS E MÉTODOS}

$\mathrm{O}$ experimento foi instalado e conduzido em área pertencente ao Departamento de Fitossanidade da Faculdade de Ciências Agrárias e Veterinárias, UNESP, no município de Jaboticabal, Estado de São Paulo. O clima da região é caracterizado por uma precipitação média anual de 1388,0 mm e as temperaturas médias anuais máximas e mínimas de 29,4 e $17,0{ }^{\circ} \mathrm{C}$, respectivamente. $\mathrm{O}$ solo do local possui classe textural média, com $33 \%$ de argila.

$\mathrm{O}$ experimento foi instalado no delineamento experimental de blocos casualizados, em três repetições. Para maior facilidade de visualização, os tratamentos estão apresentados na Tabela 01. As parcelas experimentais constaram de cinco linhas espaçadas de $0,9 \mathrm{~m}$ e com 6,0 
metros de comprimento, perfazendo área de $27 \mathrm{~m}^{2}$. Como área útil foram consideradas as três linhas centrais.

Os herbicidas foram aplicados em pós-emergência precoce das plantas daninhas e da

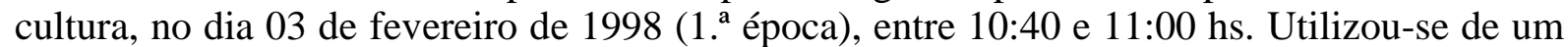
pulverizador costal manual, à pressão constante (mantida por ar comprimido) de $2,8 \mathrm{~kg} / \mathrm{cm}^{2}$, munido de barra com 4 bicos de jato plano (tipo "leque") XR 11002 VS e consumo de calda equivalente a $200 \mathrm{~L} / \mathrm{ha}$. Por ocasião da aplicação, o solo encontrava-se com boa umidade, a temperatura ambiente $30^{\circ} \mathrm{C}$, a temperatura do solo (a $5 \mathrm{~cm}$ de profundidade) $33^{\circ} \mathrm{C}$, e umidade relativa do ar de $70 \%$, os ventos intermitentes e sem prejuízos para a aplicação e com nebulosidade de 40\%. O híbrido utilizado foi o AG 3010 (Agroceres) e a adubação de manutenção foi feita com $250 \mathrm{Kg} / \mathrm{ha}$ da fórmula 4-20-20.

$\mathrm{Na}$ ocasião da primeira aplicação as plantas avaliadas apresentavam-se nos seguintes estágios: a cultura encontrava-se com 4 a 7 folhas, capim-carrapicho (Cenchrus echinatus) com 2 a 3 folhas; capim colchão (Digitaria horizontalis), beldroega (Portulaca oleracea) com 2 folhas, anileira (Indigofera hirsuta) e corda-de-viola (Ipomoea grandifolia) com 2 folhas.

A aplicação em pós emergência semi-tardia foi realizada no dia 10 de fevereiro (2. ${ }^{a}$ época), entre 17:20 e 17:50 hs. As variações com relação a aplicação da 1. ${ }^{\text {a }}$ época foram: temperatura ambiente $31^{\circ} \mathrm{C}$, a temperatura do solo (a $5 \mathrm{~cm}$ de profundidade) $37^{\circ} \mathrm{C}$, e umidade relativa do ar de $75 \%$ com nebulosidade de $90 \%$ e solo seco na superfície. O curto intervalo entre as aplicações deu-se devido ao maior crescimento da cultura em relação as plantas daninhas, sendo que o milho poderia promover efeito "guarda-chuva" se a aplicação fosse retardada. Para esta época de aplicação o milho apresentava-se com 8 a 10 folhas e as plantas daninhas descritas com 4 a 5 folhas, exceto a beldroega que apresentava-se com 8 a 10 folhas.

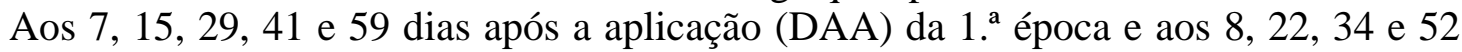

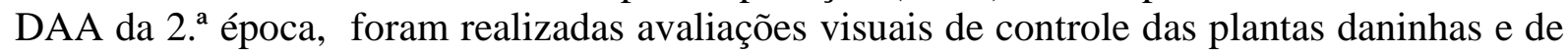
fitotoxicidade para observação de possíveis alterações morfológicas que pudessem ser caracterizadas como efeitos da intoxicação pelos herbicidas. As notas de fitotoxicidade foram baseadas na escala proposta dentro dos procedimentos para instalação, avaliação e análise de experimentos com herbicidas (SBCPD, 1995).

Para avaliações dos níveis de eficácia dos herbicidas utilizados, atribuiuse, visualmente, porcentagens de controle. Aos 125 DAA, em relação à primeira aplicação, na ocasião da colheita, foi efetuada a contagem de plantas na área útil e obtidos os resultados de produção de grãos.

\section{RESULTADOS E DISCUSSÃO}

As principais plantas daninhas que ocorreram no ensaio e suas respectivas porcentagens de infestação foram: capim-carrapicho (Cenchrus echinatus) 25\%; capimcolchão (Digitaria horizontalis) 20\%; beldroega (Portulaca oleracea) 15\%; anileira (Indigofera hirsuta) $20 \%$ e corda-de-viola (Ipomoea grandifolia) $10 \%$.

A adição de acetochlor proporcionou melhores índices de controle em Cenchrus echinatus, independente da dose testada (Tabela 02). No entanto, verifica-se que retardando a aplicação em uma semana, ganha-se em residual garantindo bons resultados, ao redor de 90 $\%$ de controle para as misturas de acetochlor com nicosulfuron, pelo fato de abrangir um maior número de plantas emergidas. Cabe ressaltar que a interferência das plantas daninhas no milho ocorre do início da emergência e estende-se por mais 15 a 25 dias segundo Blanco et al. (1976) e Pitelli (1985), citados por Zagonel et al. (2000). Dessa forma, a aplicação realizada mais precocemente torna-se favorável. Merotto Junior et al. (1997) ratificam essa proposta, onde constataram que aplicações de herbicidas no controle de Brachiaria 
plantaginea na fase inicial de desenvolvimento do milho proporcionou maior rendimento de grãos em relação as aplicações realizadas após 28 dias da semeadura.

Em relação a época de aplicação nota-se que para as demais plantas daninhas avaliadas no experimento, a aplicação dos herbicidas realizadas mais precocemente proporcionou melhora nos seus respectivos controles. Contrastando com esse resultado Barros et al. (2000) não verificaram diferenças marcantes entre as épocas de controle de Ipomoea grandifolia e Euphorbia heterophyla para os herbicidas testados, excetuando-se o controle de Commelina benghalensis que foi deficiente na aplicação do nicosulfuron em pós-emergência avançada.

A adição do acetochlor proporcionou melhor controle de Digitaria horizontalis e Indigofera hirsuta, principalmente em pós-emergência precoce (Tabelas 03 e 04), não havendo efeito da dose. Segundo Rabaey \& Harvey (1997), o acetochlor demonstrou melhora no controle de Eriochloa villosa e Panicum miliaceum, quando associado com nicosulfuron. $\mathrm{O}$ nicosulfuron, aplicado isoladamente, demonstrou uma rápida e crescente perda na sua eficácia de controle dessas plantas daninhas. No entanto, a $D$. horizontalis foi mais facilmente controlada em todas as dosagens testadas da mistura do que a I. hirsuta que apresentou uma queda expressiva no seu controle com índices ao redor de $66 \%$ nas menores doses $(1,68$ e $2,52 / 0,032 \mathrm{~kg} / \mathrm{ha}$ ) da mistura de acetochlor com nicosulfuron, a partir do 29 DAA. O controle de $D$. horizontalis manteve-se suficiente $(80 \%)$ até os 59 DAA, não diferindo estatisticamente da testemunha capinada.

As plantas daninhas Ipomoea grandifolia e Portulaca oleracea foram eficientemente controladas apenas pelo nicosulfuron na aplicação precoce, garantindo níveis de controle acima de $95 \%$ até os 59 DAA como ilustram as Tabelas 05 e 06 . No entanto, quando adicionou-se o acetochlor, nas doses 1,68 e $2,52 \mathrm{~kg} / \mathrm{ha}$ ao nicosulfuron observou-se que algumas plantas de $I$. grandifolia rebrotaram. Brighenti et al. (2000) verificaram que o controle de $I$. grandifoloia não foi satisfatório(89\%), deido a importância desta planta daninha na cultura, quando utilizou-se o acetochlor a $2,5 \mathrm{~kg} / \mathrm{ha}$ e que reduzindo-se a dose para 2,1 $\mathrm{kg} / \mathrm{ha}$ acentuou a queda no controle para $37 \%$.

Os herbicidas podem causar diferentes graus de injúrias de acordo com a espécie e o estádio das plantas daninhas. Também, características inerentes aos herbicidas podem afetar o nível de controle, tais como dosagens diferentes, misturas com outros herbicidas e adição de adjuvantes. Santos (1998) exemplifica muito bem, relatando que o capim-carrapicho é bem suscetível ao nicosulfuron a $0,4 \mathrm{~kg} / \mathrm{ha}$, o apaga-fogo é eficazmente controlado pelo nicosulfuron a 0,$2 ; 0,25 ; 0,3$ e $0,4 \mathrm{~kg} / \mathrm{ha}$ desde que aplicado em mistura com atrazina mais óleo mineral a 1,2 / 0,9 e a 2,4/1,8 kg/ha, respectivamente. No entanto, a trapoeraba não é controlada pelo nicosulfuron em nenhuma das doses testadas.

Uma leve clorose no meio da folha do milho e algum enrolamento das mesmas foi notado quando adicionou-se o acetochlor na aplicação em pós-emergência precoce. Nesta ocasião o milho encontrava-se com 4 a 7 folhas. Porém, 15 dias após a aplicação não notou-se diferenças visíveis entre as plantas nos tratamentos. No entanto, o nicosulfuron pode causar alguma injúria às plantas de milho, que segundo Môro et al. (1999) observaram descolorações da lâmina foliar central em alguns híbridos de milho aos 7 dias após a aplicação do nicosulfuron à $80 \mathrm{~g} / \mathrm{ha}$. Os demais tratamentos apresentaram produção intermediária entre as testemunhas, devido ao controle deficiente de algumas espécies daninhas. Merotto Junior et al. (1997) destacaram que a sensibilidade do rendimento de grãos foi mais afetada pela competição causada pelas plantas daninhas. $\mathrm{O}$ tratamento com acetochlor utilizado na maior dose (3,36/0,032 kg i.a./ha), aplicado tardiamente, proporcionou um rendimento de grãos significativamente menor em relação aos demais tratamentos. Brighenti et al. (2000) também observaram que o acetochlor a $2,52 \mathrm{~kg} / \mathrm{ha}$ ocasionou porcentagens de intoxicação ao girassol da ordem de $25 \%$. 


\section{CONCLUSÃO}

Excetuando-se o Cenchrus echinatus que foi melhor controlado tardiamente, os controles das demais plantas daninhas foram mais eficazes na aplicação precoce dos herbicidas, devido à maior suscetibilidade dessas espécies na fase inicial do seu desenvolvimento ao nicosulfuron.

O aumento da dose do acetochlor de 1,68 até 3,36 kg/ha nas misturas, não proporcionou melhora no controle das plantas daninhas, ocasionando apenas redução no rendimento de grãos do milho, quando aplicado na maior dose $(3,36 \mathrm{~kg} / \mathrm{ha})$ em mistura com nicosulfuron a $0,032 \mathrm{~kg} / \mathrm{ha}$, tardiamente.

\section{LITERATURA CITADA}

AGRIANUAL 2000: Milho - Anuário da Agricultura Brasileira. São Paulo : FNP/ Consultoria \& Comércio, 1999. 435p.

BARROS, A.C.; UEDA, A.; SCHUMM, K.C. Efeito de herbicidas de pós-emergência, aplicados em várias épocas, comparados com atrazine+metolachlor, em pré-emergência, na cultura do milho. Revista Brasileira de Herbicidas, v.1, n.3, p. 207-212, 2000.

BLANCO, H.G. Ecologia das plantas daninhas - competição de plantas daninhas em culturas brasileiras. In: CONTROLE INTEGRADO DE PLANTAS DANINHAS: 2 ed. São Paulo: CREA, 1985. 131p.

BRIGHENTI, A.M.; GAZZIERO, D.L.P; OLIVEIRA, M.F.; VOLL, E.; PEREIRA, J.E. Controle químico de plantas daninhas na cultura do girassol em solo de textura argilosa. Revista Brasileira de Herbicidas, v.1, n.1, p. 85-88, 2000.

CARVALHO, F.T.; PERUCHI, M.; PALAZZO, R.R.B. Eficácia de herbicidas no controle, em pós-emergência, de plantas daninhas na cultura do milho. Revista Brasileira de Herbicidas, v.2, n.3, p. 143-147, 2001.

CHRISTOFFOLETI, P.J.; VICTORIA FILHO, R.; SILVA, C.B. Resistência de plantas daninhas aos herbicidas. Planta Daninha, v.12, n.1, p. 13-20, 1994.

GAZZIERO, D.L.P.; ADEGAS, F.S.; PRETE, C.E.C.; RALISCH, R.; GUIMARÃES, M.F. As plantas daninhas e a semeadura direta. Londrina, PR, 2001. 59p. (EMBRAPA SOJA, Cirular Técnica, 33).

MEROTTO JUNIOR, A.; GUIDOLIN, A.F.; ALMEIDA, M.L.; HARVERROTH, H.S. Aumento da população de plantas e uso de herbicidas no controle de plantas daninhas em milho. Planta Daninha, v.15, n.12, p. 141-151, 1997.

MONQUERO, P.A.; CHRISTOFFOLETI, P.J. Manejo de populações de plantas daninhas resistentes aos herbicidas inibidores da acetolactato sintase. Planta Daninha, v.19, n.1, p. 67-74, 2001. 
MÔRO, F.V.; DAMINHÃ̃ FILHO, C.F. Alterações morfo-anatômicas das folhas de milho submetidas à aplicação de nicosulfuron. Planta Daninha, v.17, n.3, p. 331-337, 1999.

RABAEY, T.L. \& HARVEY, R.G. Sequential applications control woolly cupgrass (Eriochloa villosa) and wild-proso millet (Panicum miliaceum) in corn (Zea mays). Weed Technology, v. 11, n. 3, p. 537-542, 1997.

SANTOS, C.A. Manejo das plantas daninhas em plantio direto e convencional, com nicosulfuron isolado e em mistura com atrazin, na cultura do milho (Zea mays L.), Jaboticabal, São Paulo, 1998. 60p. Monografia (Trabalho de graduação em Agronomia) - Faculdade de Ciências Agrárias e Veterinárias, Universidade Estadual Paulista, Jaboticabal 1998.

SOCIEDADE BRASILEIRA DA CIÊNCIA DAS PLANTAS DANINHAS (SBCPD). Procedimentos para instalação, avaliação e análise de experimentos com herbicidas. Londrina, 1995. 42p.

ZAGONEL, J.; VENÂNCIO, W.S.; KUNZ, R.P. Efeitos de métodos e épocas de controle das plantas daninhas na cultura do milho. Planta Daninha, v.18, n.1, p. 143-150, 2000.

Tabela 01 - Tratamentos avaliados na cultura do milho (AG 3010). Jaboticabal, SP. 1998. 


\begin{tabular}{lcccc}
\hline \multirow{2}{*}{ Tratamentos } & \multirow{2}{*}{ Nome comercial } & \multirow{2}{*}{$\begin{array}{c}\text { Época de } \\
\text { aplicação }\end{array}$} & \multicolumn{2}{c}{ Doses } \\
\cline { 5 - 6 } & & I.A. ${ }^{1}(\mathrm{~kg} / \mathrm{ha})$ & P.C. ${ }^{2}$ (L/ha) \\
\hline 1. acetochlor / nicosulfuron & Kadett / Sanson & pós precoce & $1,68 / 0,032$ & $2,0 / 0,8$ \\
2. acetochlor / nicosulfuron & Kadett / Sanson & pós precoce & $2,52 / 0,032$ & $3,0 / 0,8$ \\
3. acetochlor / nicosulfuron & Kadett / Sanson & pós precoce & $3,36 / 0,032$ & $4,0 / 0,8$ \\
4. acetochlor / nicosulfuron & Kadett / Sanson & pós tardia & $1,68 / 0,032$ & $2,0 / 0,8$ \\
5. acetochlor / nicosulfuron & Kadett / Sanson & pós tardia & $2,52 / 0,032$ & $3,0 / 0,8$ \\
6. acetochlor / nicosulfuron & Kadett / Sanson & pós tardia & $3,36 / 0,032$ & $4,0 / 0,8$ \\
7. nicosulfuron & Sanson & pós precoce & 0,032 & 0,8 \\
8. nicosulfuron & Sanson & pós tardia & 0,032 & 0,8 \\
9. testemunha sem capina & - & - & - & - \\
10.testemunha capinada & - & - & - & - \\
1. Ingrediente ativo & & & & \\
2 Produto comercial & & & &
\end{tabular}


Tabela 02 - Médias das percentagens de controle, atribuídas visualmente, para Cenchrus echinatus na cultura do milho, em diferentes épocas após a aplicação dos herbicidas, em pós-emergência. Jaboticabal-SP. 1998.

\begin{tabular}{|c|c|c|c|c|c|c|c|}
\hline \multirow{3}{*}{ Tratamentos } & \multirow{3}{*}{$\begin{array}{c}\text { Doses } \\
\text { I.A. }(\mathrm{kg} / \mathrm{ha})\end{array}$} & \multirow{3}{*}{$\begin{array}{l}\text { Aplicação } \\
\text { 1. }{ }^{a} \text { época } \\
\text { 2. }{ }^{a} \text { época }\end{array}$} & \multicolumn{5}{|c|}{ Controle $(\%)$} \\
\hline & & & $07^{1} \mathrm{DAA}$ & $15 \mathrm{DAA}$ & 29 DAA & 44 DAA & 59 DAA \\
\hline & & & - & $08^{2} \mathrm{DAA}$ & 22 DAA & 34 DAA & 52 DAA \\
\hline 1. acetochlor / nicosulfuron & $1,68 / 0,032$ & PR & $98,0^{3} \mathrm{ab}^{4}$ & $99,0 \mathrm{a}$ & $96,0 \mathrm{ab}$ & $68,3 \mathrm{bc}$ & $70,0 \mathrm{bc}$ \\
\hline 2. acetochlor / nicosulfuron & $2,52 / 0,032$ & PR & 99,3 a & $99,0 \mathrm{a}$ & $97,7 \mathrm{a}$ & $76,7 \mathrm{bc}$ & $78,3 \mathrm{bc}$ \\
\hline 3. acetochlor / nicosulfuron & $3,36 / 0,032$ & PR & $99,0 \mathrm{a}$ & $97,0 \mathrm{ab}$ & $97,0 \mathrm{a}$ & $86,7 \mathrm{abc}$ & $81,7 \mathrm{bc}$ \\
\hline 4. acetochlor / nicosulfuron & $1,68 / 0,032$ & TA & - & $94,3 \mathrm{abc}$ & $98,0 \mathrm{a}$ & $93,3 \mathrm{ab}$ & $91,7 \mathrm{ab}$ \\
\hline 5. acetochlor / nicosulfuron & $2,52 / 0,032$ & TA & - & $96,7 \mathrm{ab}$ & $97,0 \mathrm{a}$ & $95,0 \mathrm{ab}$ & $91,7 \mathrm{ab}$ \\
\hline 6. acetochlor / nicosulfuron & $3,36 / 0,032$ & $\mathrm{TA}$ & - & 99,0 a & $99,0 \mathrm{a}$ & $95,0 \mathrm{ab}$ & $90,0 \mathrm{ab}$ \\
\hline 7. nicosulfuron & 0,032 & PR & $88,3 \mathrm{~b}$ & $73,3 \quad c$ & $56,7 \quad c$ & $56,7 \quad \mathrm{c}$ & $56,7 \quad c$ \\
\hline 8.nicosulfuron & 0,032 & TA & - & $83,3 \mathrm{bc}$ & $83,3 \mathrm{~b}$ & $86,7 \mathrm{abc}$ & $83,3 \mathrm{bc}$ \\
\hline 9. testemunha sem capina & - & - & $(26,7)^{5}$ & $(30,0)$ & $(23,3)$ & $(21,7)$ & $(15,0)$ \\
\hline 10.testemunha capinada & - & - & $100,0 \mathrm{a}$ & 100,0 a & $100,0 \mathrm{a}$ & $100,0 \mathrm{a}$ & $100,0 \mathrm{a}$ \\
\hline $\mathrm{CV}(\%)$ & & & 10,6 & 8,6 & 5,8 & 11,7 & 11,5 \\
\hline
\end{tabular}

${ }^{1}$ Dias após a primeira aplicação

${ }^{2}$ Dias pós a segunda aplicação

3 Dados originais

${ }^{4}$ Análise estatística feita com dados transformados em arc sen $\sqrt{ } \%$

${ }^{5}$ Porcentagem de cobertura do solo original

DAA - Dias Após a Aplicação

PR - pós precoce

TA - pós tardia 
Tabela 03 - Médias das percentagens de controle, atribuídas visualmente, para Digitaria horizontalis na cultura do milho, em diferentes épocas após a aplicação dos herbicidas, em pós-emergência. Jaboticabal-SP. 1998.

\begin{tabular}{|c|c|c|c|c|c|c|c|}
\hline \multirow{3}{*}{ Tratamentos } & \multirow{3}{*}{$\begin{array}{c}\text { Doses } \\
\text { I.A. }(\mathrm{kg} / \mathrm{ha})\end{array}$} & \multirow{3}{*}{$\begin{array}{l}\text { Aplicação } \\
\text { 1. a época } \\
\text { 2. }{ }^{\mathrm{a}} \text { época }\end{array}$} & \multicolumn{5}{|c|}{ Controle $(\%)$} \\
\hline & & & $07^{1} \mathrm{DAA}$ & 15 DAA & 29 DAA & 44 DAA & 59 DAA \\
\hline & & & - & $08^{2} \mathrm{DAA}$ & $22 \mathrm{DAA}$ & 34 DAA & 52 DAA \\
\hline 1. acetochlor / nicosulfuron & $1,68 / 0,032$ & PR & $100,0^{3} \mathrm{a}^{4}$ & $100,0 \mathrm{a}$ & $93,3 \mathrm{ab}$ & $88,3 \mathrm{ab}$ & $80,0 \mathrm{ab}$ \\
\hline 2. acetochlor / nicosulfuron & $2,52 / 0,032$ & PR & $100,0 \mathrm{a}$ & $98,7 \mathrm{ab}$ & $91,7 \mathrm{ab}$ & $88,3 \mathrm{ab}$ & $85,0 \mathrm{ab}$ \\
\hline 3. acetochlor / nicosulfuron & $3,36 / 0,032$ & PR & $100,0 \mathrm{a}$ & 99,3 a & $92,7 \mathrm{ab}$ & $85,0 \mathrm{ab}$ & $81,7 \mathrm{ab}$ \\
\hline 4. acetochlor / nicosulfuron & $1,68 / 0,032$ & TA & - & $82,7 \mathrm{ab}$ & 63,3 bc & 46,7 bc & $41,7 \mathrm{bcd}$ \\
\hline 5. acetochlor / nicosulfuron & $2,52 / 0,032$ & TA & - & $85,0 \mathrm{ab}$ & $66,7 \mathrm{~b}$ & $66,7 \mathrm{abc}$ & $50,0 \mathrm{abc}$ \\
\hline 6. acetochlor / nicosulfuron & $3,36 / 0,032$ & TA & - & $90,0 \mathrm{ab}$ & 53,3 bcd & $63,3 \mathrm{bc}$ & 46,7 bcd \\
\hline 7. nicosulfuron & 0,032 & PR & $98,3 \quad b$ & 63,3 bc & 21,7 & 20,0 & 11,7 \\
\hline 8.nicosulfuron & 0,032 & TA & - & $26,7 \quad c$ & 18,3 & 16,7 & 18,3 \\
\hline 9. testemunha sm capina & - & - & $(20,0)^{5}$ & $(15,0)$ & $(18,3)$ & $(21,7)$ & $(20,0)$ \\
\hline 10.testemunha capinada & - & - & $100,0 \mathrm{a}$ & $100,0 \mathrm{a}$ & $100,0 \mathrm{a}$ & $100,0 \mathrm{a}$ & $100,0 \mathrm{a}$ \\
\hline $\mathrm{CV}(\%)$ & & & 5,3 & 14,3 & 18,5 & 23,9 & 25,4 \\
\hline
\end{tabular}

${ }^{1}$ Dias após a primeira aplicação

${ }^{2}$ Dias pós a segunda aplicação

${ }^{3}$ Dados originais

${ }^{4}$ Análise estatística feita com dados transformados em arc sen $\sqrt{ } \%$

${ }^{5}$ Porcentagem de cobertura do solo original

DAA - Dias Após a Aplicação

PR - pós precoce

TA - pós tardia 
Tabela 04 - Médias das percentagens de controle, atribuídas visualmente, para Indigofera hirsuta na cultura do milho, em diferentes épocas após a aplicação dos herbicidas, em pós-emergência. Jaboticabal-SP. 1998.

\begin{tabular}{|c|c|c|c|c|c|c|c|}
\hline \multirow{3}{*}{ Tratamentos } & \multirow{3}{*}{$\begin{array}{c}\text { Doses } \\
\text { I.A. }(\mathrm{kg} / \mathrm{ha})\end{array}$} & Aplicação & \multicolumn{5}{|c|}{ Controle $(\%)$} \\
\hline & & 1. época & $07^{1} \mathrm{DAA}$ & $15 \mathrm{DAA}$ & $29 \mathrm{DAA}$ & 44 DAA & 59 DAA \\
\hline & & 2. época & - & $08^{2} \mathrm{DAA}$ & 22 DAA & 34 DAA & 52 DAA \\
\hline 1. acetochlor / nicosulfuron & $1,68 / 0,032$ & PR & $83,3^{3} \mathrm{bc}^{4}$ & $90,0 \mathrm{~b}$ & $66,7 \mathrm{bc}$ & $43,3 \mathrm{~b}$ & 8,3 bcd \\
\hline 2. acetochlor / nicosulfuron & $2,52 / 0,032$ & PR & $85,0 \mathrm{~b}$ & $90,0 \mathrm{~b}$ & $66,7 \mathrm{bc}$ & $36,7 \mathrm{bc}$ & 10,0 bc \\
\hline 3. acetochlor / nicosulfuron & $3,36 / 0,032$ & PR & $86,7 \mathrm{~b}$ & $91,7 \mathrm{~b}$ & $73,3 \mathrm{~b}$ & $50,0 \mathrm{~b}$ & $21,7 \quad b$ \\
\hline 4. acetochlor / nicosulfuron & $1,68 / 0,032$ & TA & - & $43,3 \quad c$ & $40,0 \mathrm{~cd}$ & $26,7 \mathrm{bcd}$ & 3,3 \\
\hline 5. acetochlor / nicosulfuron & $2,52 / 0,032$ & TA & - & 53,3 c & $50,0 \mathrm{bc}$ & $33,3 \mathrm{bc}$ & $10,0 \mathrm{bc}$ \\
\hline 6. acetochlor / nicosulfuron & $3,36 / 0,032$ & TA & - & $46,7 \quad c$ & $43,3 \mathrm{~cd}$ & $30,0 \mathrm{bc}$ & 5,0 bcd \\
\hline 7. nicosulfuron & 0,032 & PR & 71,7 & $36,7 \mathrm{~cd}$ & 20,0 & $13,3 \mathrm{~cd}$ & 1,7 \\
\hline 8.nicosulfuron & 0,032 & TA & - & 16,7 & 8,3 & 6,7 & 0,0 \\
\hline 9. testemunha sem capina & - & - & $(20,0)^{5}$ & $(16,7)$ & $(21,7)$ & $(20,0)$ & $(30,0)$ \\
\hline 10.testemunha capinada & - & - & $100,0 \mathrm{a}$ & $100,0 \mathrm{a}$ & $100,0 \mathrm{a}$ & $100,0 \mathrm{a}$ & $100,0 \mathrm{a}$ \\
\hline $\mathrm{CV}(\%)$ & & & 8,9 & 2,8 & 13,7 & 15,8 & 31,1 \\
\hline
\end{tabular}

${ }^{1}$ Dias após a primeira aplicação

${ }^{2}$ Dias pós a segunda aplicação

${ }^{3}$ Dados originais

${ }^{4}$ Análise estatística feita com dados transformados em arc sen $\sqrt{ } \%$

${ }^{5}$ Porcentagem de cobertura do solo original

DAA - Dias Após a Aplicação

PR - pós precoce

TA - pós tardia 
Tabela 05 - Médias das percentagens de controle, atribuídas visualmente, para Portulaca oleracea na cultura do milho, em diferentes épocas após a aplicação dos herbicidas, em pós-emergência. Jaboticabal-SP. 1998.

\begin{tabular}{|c|c|c|c|c|c|c|c|}
\hline \multirow{3}{*}{ Tratamentos } & \multirow{3}{*}{$\begin{array}{c}\text { Doses } \\
\text { I.A. }(\mathrm{kg} / \mathrm{ha})\end{array}$} & \multirow{3}{*}{$\begin{array}{l}\text { Aplicação } \\
\text { 1. a época } \\
\text { 2. a época }\end{array}$} & \multicolumn{5}{|c|}{ Controle $(\%)$} \\
\hline & & & $07^{1} \mathrm{DAA}$ & $15 \mathrm{DAA}$ & 29 DAA & 44 DAA & 59 DAA \\
\hline & & & - & $08^{2} \mathrm{DAA}$ & 22 DAA & 34 DAA & 52 DAA \\
\hline 1. acetochlor / nicosulfuron & $1,68 / 0,032$ & PR & $100,0^{3} \mathrm{a}^{4}$ & $100,0 \mathrm{a}$ & $100,0 \mathrm{a}$ & $96,7 \mathrm{ab}$ & $96,7 \mathrm{a}$ \\
\hline 2. acetochlor / nicosulfuron & $2,52 / 0,032$ & PR & $100,0 \mathrm{a}$ & $100,0 \mathrm{a}$ & $100,0 \mathrm{a}$ & $95,0 \mathrm{ab}$ & $95,0 \mathrm{a}$ \\
\hline 3. acetochlor / nicosulfuron & $3,36 / 0,032$ & PR & $100,0 \mathrm{a}$ & $100,0 \mathrm{a}$ & $100,0 \mathrm{a}$ & $95,7 \mathrm{ab}$ & $95,7 \mathrm{a}$ \\
\hline 4. acetochlor / nicosulfuron & $1,68 / 0,032$ & TA & - & $46,7 \quad b$ & $56,7 \mathrm{ab}$ & $56,7 \mathrm{abc}$ & $50,0 \mathrm{ab}$ \\
\hline 5. acetochlor / nicosulfuron & $2,52 / 0,032$ & TA & - & $55,0 \quad b$ & $63,3 \mathrm{ab}$ & 50,0 bc & $40,0 \mathrm{ab}$ \\
\hline 6. acetochlor / nicosulfuron & $3,36 / 0,032$ & TA & - & $53,3 \quad b$ & $56,7 \mathrm{ab}$ & 53,3 bc & $53,3 \mathrm{ab}$ \\
\hline 7. nicosulfuron & 0,032 & PR & $100,0 \mathrm{a}$ & $95,0 \mathrm{a}$ & $90,0 \mathrm{ab}$ & $95,0 \mathrm{ab}$ & $95,0 \mathrm{a}$ \\
\hline 8.nicosulfuron & 0,032 & TA & - & 10,0 & $7,5 \mathrm{~b}$ & 10,0 & $6,7 b$ \\
\hline 9. testemunha infestada & - & - & $(15,0)^{5}$ & $(16,7)$ & $(10,0)$ & $(10,0)$ & $(5,0)$ \\
\hline 10.testemunha capinada & - & - & $100,0 \mathrm{a}$ & $100,0 \mathrm{a}$ & $100,0 \mathrm{a}$ & $100,0 \mathrm{a}$ & $100,0 \mathrm{a}$ \\
\hline $\mathrm{CV}(\%)$ & & & 0,0 & 7,3 & 23,7 & 21,2 & 23,1 \\
\hline
\end{tabular}

${ }^{1}$ Dias após a primeira aplicação

${ }^{2}$ Dias pós a segunda aplicação

${ }^{3}$ Dados originais

${ }^{4}$ Análise estatística feita com dados transformados em arc sen $\sqrt{ } \%$

${ }^{5}$ Porcentagem de cobertura do solo original

DAA - Dias Após a Aplicação

PR - pós precoce

TA - pós tardia 
Tabela 06 - Médias das percentagens de controle, atribuídas visualmente, para Ipomoea grandifolia na cultura do milho, em diferentes épocas após a aplicação dos herbicidas, em pós-emergência. Jaboticabal-SP. 1998.

\begin{tabular}{|c|c|c|c|c|c|c|c|}
\hline \multirow{3}{*}{ Tratamentos } & \multirow{3}{*}{$\begin{array}{c}\text { Doses } \\
\text { I.A. }(\mathrm{kg} / \mathrm{ha})\end{array}$} & Aplicação & \multicolumn{5}{|c|}{ Controle $(\%)$} \\
\hline & & 1. ápoca & $07^{1} \mathrm{DAA}$ & $15 \mathrm{DAA}$ & $29 \mathrm{DAA}$ & 44 DAA & 59 DAA \\
\hline & & 2. ${ }^{\mathrm{a}}$ época & - & $08^{2} \mathrm{DAA}$ & 22 DAA & 34 DAA & 52 DAA \\
\hline 1. acetochlor / nicosulfuron & $1,68 / 0,032$ & PR & $76,7^{3} b^{4}$ & $90,0 \mathrm{ab}$ & $100,0 \mathrm{a}$ & $96,7 \mathrm{a}$ & $96,7 \mathrm{ab}$ \\
\hline 2. acetochlor / nicosulfuron & $2,52 / 0,032$ & PR & 80,0 b & $90,0 \mathrm{ab}$ & $100,0 \mathrm{a}$ & $93,3 \mathrm{a}$ & 93,3 abc \\
\hline 3. acetochlor / nicosulfuron & $3,36 / 0,032$ & PR & $81,7 \mathrm{~b}$ & $100,0 \mathrm{a}$ & $100,0 \mathrm{a}$ & $100,0 \mathrm{a}$ & $100,0 \mathrm{a}$ \\
\hline 4. acetochlor / nicosulfuron & $1,68 / 0,032$ & TA & - & 73,3 abc & $80,0 \mathrm{ab}$ & $73,3 \mathrm{ab}$ & 73,3 abc \\
\hline 5. acetochlor / nicosulfuron & $2,52 / 0,032$ & TA & - & $83,3 \mathrm{abc}$ & $100,0 \mathrm{a}$ & 96,7 a & $86,7 \mathrm{ab}$ \\
\hline 6. acetochlor / nicosulfuron & $3,36 / 0,032$ & TA & - & 80,0 bc & $90,0 \mathrm{ab}$ & $80,0 \mathrm{ab}$ & 73,3 abc \\
\hline 7. nicosulfuron & 0,032 & PR & $83,3 \mathrm{~b}$ & $100,0 \mathrm{a}$ & $100,0 \mathrm{a}$ & $100,0 \mathrm{a}$ & $100,0 \mathrm{a}$ \\
\hline 8.nicosulfuron & 0,032 & TA & - & 43,3 & $66,7 \quad b$ & $46,7 \quad b$ & 46,7 \\
\hline 9. testemunha sem capina & - & - & $(8,3)^{5}$ & $(5,0)$ & $(5,0)$ & $(6,7)$ & $(6,7)$ \\
\hline 10.testemunha capinada & - & - & $100,0 \mathrm{a}$ & $100,0 \mathrm{a}$ & $100,0 \mathrm{a}$ & $100,0 \mathrm{a}$ & $100,0 \mathrm{a}$ \\
\hline $\mathrm{CV}(\%)$ & & & 13,5 & 19,7 & 14,8 & 16,8 & 17,9 \\
\hline
\end{tabular}

${ }^{1}$ Dias após a primeira aplicação

${ }^{2}$ Dias pós a segunda aplicação

${ }^{3}$ Dados originais

${ }^{4}$ Análise estatística feita com dados transformados em arc sen $\sqrt{ } \%$

${ }^{5}$ Porcentagem de cobertura do solo original

DAA - Dias Após a Aplicação

PR - pós precoce

TA - pós tardia 
Tabela 07 - Produção média de grãos do milho híbrido AG 3010. Jaboticabal - SP, 1998.

\begin{tabular}{lccc}
\hline \multicolumn{1}{c}{ Tratamentos } & $\begin{array}{c}\text { Doses } \\
\text { I.A (kg/ha) }\end{array}$ & $\begin{array}{c}\text { Época da } \\
\text { aplicação }\end{array}$ & $\begin{array}{c}\text { Produção de grãos } \\
\text { (gramas) em 15m² }\end{array}$ \\
\hline 1. acetochlor / nicosulfuron & $1,68 / 0,032$ & PR & $3260,0 \mathrm{ab}$ \\
2. acetochlor / nicosulfuron & $2,52 / 0,032$ & PR & $2926,7 \mathrm{ab}$ \\
3. acetochlor / nicosulfuron & $3,36 / 0,032$ & PR & $3566,7 \mathrm{ab}$ \\
4. acetochlor / nicosulfuron & $1,68 / 0,032$ & TA & $2833,3 \mathrm{ab}$ \\
5. acetochlor / nicosulfuron & $2,52 / 0,032$ & TA & $3056,7 \mathrm{ab}$ \\
6. acetochlor / nicosulfuron & $3,36 / 0,032$ & TA & $2576,7 \mathrm{~b}$ \\
7. nicosulfuron & 0,032 & PR & $3576,7 \mathrm{ab}$ \\
8.nicosulfuron & 0,032 & TA & $3523,3 \mathrm{ab}$ \\
9. testemunha infestada & - & - & $2593,3 \mathrm{~b}$ \\
10.testemunha capinada & - & - & $4086,7 \mathrm{a}$ \\
\hline CV $(\%)$ & & & 14,3 \\
dms $(1 \%)$ & & & 1337,9 \\
\hline PR & &
\end{tabular}

PR - pós precoce

TA - pós tardia 